\title{
Microscopie en rayons X-mous : techniques et performances
}

\section{Joyeux}

\section{Institut d'Optique, BP. 147, 91403 Orsay Cedex, France}

\section{I - INTRODUGTION}

L'objet de cet article est de présenter le domaine la microscopie en X mous à haute résolution, en particulier sous l'angle des conditions de mise en oeuvre et d'exploitation. Les méthodes dont il va être question sont applicables aux longueurs d'onde comprises en gros entre $10 \mathrm{~nm}$ et $2,5 \mathrm{~nm}$ (de $120 \mathrm{eV}$ à $500 \mathrm{eV}$ ), étant entendu que ces limites peuvent être débordées, surtout vers les grandes longueurs d'onde. Par haute résolution, on entend une résolution allant de quelques microns à $0,03 \mu \mathrm{m}$ dans le meilleur cas.

Avant d'entrer dans le vif du sujet, il n'est pas inutile de rappeller ce qui caractérise une imagerie. En effet, il est extrêmement courant d'entendre qu'une méthode d'imagerie est complètement caractérisée par sa résolution, quand ce n'est pas par son grandissement (voire "grossissement"), paramètre en réalité accessoire! En fait, le couple résolution/champ n'est que l'un de trois éléments fondamentaux qui caractérise une imagerie: c'est celui qui définit, hors de tout contexte d'utilisation, la qualité "technique" du dispositif d'imagerie. Mais dans la pratique, l'image est obtenue dans un contexte donné, pour un but précis, et les deux aspects suivants sont tout aussi importants:

les informations apportées, c'est à dire le paramètre objet qui est visualisé, ainsi que la nature du signal image, c'est à dire le paramètre physique - amplitude, phase d'une onde, émission secondaire, etc - qui véhicule le signal dans le système d'imagerie.

les conditions de mise en oeuvre, sont un élément tout à fait fondamental dans le contexte $X$, bien que rarement pris en compte au même niveau que les autres. Il s'agit entre autres de la nécessaire préparation de 1'objet (biologie), avec la question sous-jacente: ce qui est vu sur l'image est-il représentatif de l'objet fonctionnel, c'est à dire non préparé ? Mais il s'agit également des contraintes de mise en oeuvre, provenant des sources (voir sect.III), des composants, et du système (précision des réglages, temps de mise en oeuvre et d'analyse, rapport signal/bruit final)...

\section{II - PROPRIETES ET INTERET DE LA MICROSCOPIE X-MOUS}

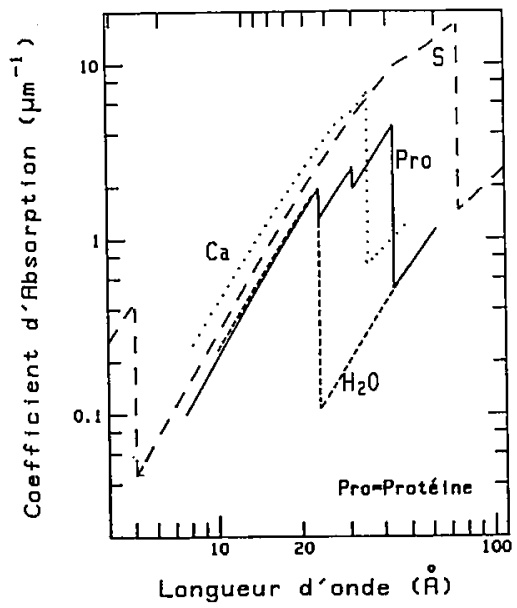

S'agissant d'imagerie à haute résolution, ce sont les biologistes qui ont d'abord reconnu l'intérêt du domaine $\mathrm{X}$ mous. La raison principale est exprimée dans le diagramme classique des coefficients d'absorption ci-contre. En effet, ce diagramme montre d'abord que la pénétration des rayons $\mathrm{X}$-mous dans la matière vivante (éléments $C, O, N$ pour l'essentiell) est adaptée aux épaisseurs des constituants "organisés" du vivant que sont la cellule et ses sous-ensembles, ou encore des systèmes comme les fibres musculaires. De pius, on voit que l'intervalle 4,4-2,5 nm présente un intérêt particulier dans la mesure ou l'eau y est beaucoup plus transparente que la matière carbonée: il est donc possible d'espérer faire l'image d'objets entiers,

Article published by EDP Sciences and available at http://www.annphys.org or http://dx.doi.org/10.1051/anphys/1992044 
non desséchés, hydratés, en un mot dans ètat très proche de l'état vivant. En fait, cette propriété s'est très récemment révélée utile dans d'autres domaines que la biologie, en particulier l'étude des gels et colloides.

Par ailleurs, les constrastes obtenus en $X$ étant liés aux éléments chimiques et dépendant de la longueur d'onde, on voit que cette imagerie dispose d'une dimension de type "couleur": l'image obtenue dépend de la longueur d'onde et de la composition chimique. En biologie, la répartition d'éléments moins abondants, mais de grande importance fonctionnelle, peut en principe être révélés (ex: Ca), et il reste toujours possible de "marquer" des molécules spécifiques pour suivre leur devenir dans la cellule. La microscopie X-mous appliquée à la biologie a donc une certaine capacité d'imagerie fonctionnelle. I'étude des matériaux peut également mettre cette propriété à profit: très récemment (1991), on a pu réaliser des séries d'images d'absorption au voisinage du seuil du carbone mettant à profit les structures de type EXAFS de façon à révéler la présence de deux polymères différents dans une matrice constituée d'un troisième.

Ces propriétés sont tout à fait différentes de ce que permet la microscopie électronique. D'une part le paramètre visualisé dans ce cas est la densité électronique; d'autre part les objets doivent subir une préparation très drastique: élimination de l'eau, découpage en tranche fine. Ce sont principalement ces différences qui fondent l'intérêt de la microscopie X mous appliquée à la biologie. Pour autant, l'application des techniques mises au point ne se limite pas à la biologie: elles peuvent être appliquées d'une manière générale à tout objet constitué d'éléments légers et d'épaisseur équivalente: des études ont ainsi été menées sur des ciments, et on peut également penser aux matériaux composites, dans la mesure où ils présentent des structures intéressantes à l'échelle de quelques 0,1 micron ou plus. De plus, les optiques $X$ employées en microscopie à balayage ont pour seule fonction de donner un spot $X$ de très petite dimension, servant de sonde d'analyse. De telles taches peuvent servir de sonde d'excitation pour 1 'étude par balayage de surfaces inhomogènes à une échelle comparable ou supérieure à la dimension de la sonde, quel que soit le signal recueilli. On peut ainsi en principe obtenir par balayage une image de transmission, mais également n'importe quelle image d'émission secondaire (cf. ci-dessous) provenant d'une surface. Cette possibilité étend l'intérèt des techniques développées pour la microscopie $X$ à balayage très en dehors de la bande 5-2,5 $\mathrm{nm}$, du coté des énergies plus faibles.

Les signaux obtenus dans un microscope traduisent I'interaction "1umière X"-matière. Le rayonnement incident est caractérisé par son amplitude, mais également par sa fréquence, et éventuellement sa polarisation. On peut distinguer deux cas.

1) Si l'on s'intéresse au signal X transmis (ou réfléchi) par l'objet, les phénomènes sont correctement décrits par l'indice complexe du milieu, c'est à dire par une absorption et un décalage de phase (ceci est d'autant plus vrai que la longueur d'onde est nettement plus grande que les distances interatomiques). On doit de plus prendre en compte la polarisation si le milieu traversé est optiquement actif, ou dans le cas d'une onde reflèchie sous forte incidence.

2) Comme on l'a mentionné, des signaux secondaires, c'est à dire de longueur d'onde ou de nature différente, peuvent également ètre engendrés: fluorescence $X$, émission d'électrons secondaires, etc.

\section{III - LES ELEMENTS D'UN HICROSGOPE X}

Un microscope ( $X$ ou autre) fait appel à un certain nombre d'éléments: source, objet, récepteur, et en général composants optiques. Leur mise en oeuvre doit respecter des règles précises, dont l'exposé complet déborderait largement du cadre de cet article. Nous ne discuterons (brièvement) 
que les points suivants:

* 1'adaptation des étendues géométriques de la source et de 1'optique, question essentielle pour caractériser correctement les sources $X$ destinées à l'imagerie;

* la nature et la caractérisation des composants optiques formateurs d'images dans le domaine X-mous.

Dans une section ultérieure, nous passerons en revue les différentes méthodes d'imagerie actuellement étudiées.

\subsection{Le couplage source-optique: étendue de source utile}

Un élement essentiel de la formation des images microscopiques est l'éclairage de l'objet: on ne peut obtenir une "bonne image" sans un éclairage correct en niveau et en nature. Ceci implique des propriétés de source adaptées à la méthode d'imagerie utilisée. Les deux propriétés de sources impliquées dans cette question sont la luminance de la source et les étendues acceptée par l'optique et émise par la source. Rappellons en d'abord les définitions:

* L'étendue géométrique est la propriété géométrique fondamentale d'un pinceau lumineux. Dans le présent contexte, il est commode de la définir par rapport à un plan objet ou image. En considérant un élément de surfa-

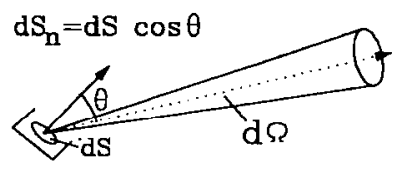
ce dS de ce plan (figure), l'étendue est le produit de l'angle solide $d \Omega$ couvert par le pinceau de rayons associés, par la surface $\mathrm{dS}_{\mathrm{n}}$ normale à ce pinseau, soit $\mathcal{E}=\mathrm{dS}_{\mathrm{n}} \mathrm{d} \Omega$. Cette quantité est caractéristique du pinceau lumineux car elle est invariante au cours de la propagation du pinceau à travers un système optique réfractif ou réflectif, en 1'absence de diaphramation (réduction d'étendue) ou de diffusion (augmentation). L'étendue $\mathrm{s}^{\prime}$ exprime en $\mathrm{m}^{2}$.steradians (ou sous-multiples).

Dans le domaine du rayonnement synchrotron, il est habituel de considérer les émittances des pinceaux, calculées pour deux sections longitudinales perpendiculaires soient $x$ et $z$. $2 \sigma$ et $2 \alpha$ étant la dimension transverse et l'angle du pinceau dans la section considérée, on utilise généralement pour 1 'émittance 1 'expression $\mathbb{E}=\sigma \alpha$ (unité pratique: mm.mrad). * la luminance d'une source est l'énergie émise par unité d'étendue et par seconde. Dans le cas où l'on utilise les emittances plutot que l'étendue, la luminance est l'énergie par unité d'émittance $x$, par unité d'émittance $z$, et par seconde.

Les propriétés de conservation de l'étendue ont plusieurs conséquences fondamentales en optique des images:

* une optique ne peut accepter une étendue supérieure à celle délimitée par son ouverture objet et son champ utile. En d'autres termes, comme aucun système optique condenseur ne peut concentrer l'énergie émise par une source dans une étendue plus faible que celle de la source, il ne sert à rien de disposer d'une source émettant dans $2 \pi$ stéradian, si 1 'ouverture optique est faible, ce qui est la situation normale en imagerie X. Pour mesurer les conséquences de cette règle incontournable, considérons une source carrée de coté $100 \mathrm{\mu m}$ émettant dans $2 \pi \mathrm{st}$. L'étendue de cette source est $6,310^{-a} \mathrm{~m}^{2} \mathrm{st}$. Considérons par ailleurs une optique limitée par la diffraction, de résolution $r=0,1 \mu \mathrm{m}$ à $\lambda=5 \mathrm{~nm}$, ayant un champ de $30 \mu \mathrm{m}$. L'étendue qu'elle accepte est $\left(30.10^{-6}\right)^{2} \times(\lambda / \mathrm{r})^{2}$ soit de $2,210^{-12} \mathrm{~m}^{2} \mathrm{st}$. Ces chiffres montrent que $\mathrm{l}^{\prime}$ énergie couplée ne peut être supérieure à $31^{-3}$ fois l'énergie émise. Il est donc tout à fait fallacieux en imagerie de comparer les sources par le flux émis, d'autant plus que dans $1^{\prime}$ exemple considéré ici, l'étendue est relativement forte.

Bien entendu, l'utilisation d'une source de forte étendue ne modifie en rien la qualité technique de l'image, dans la mesure où la puissance 
de la source est telle que l'éclairement image reste suffisant. Malheureusement, cette situation idéale $n^{\prime}$ est pas générale en microscopie $X$ : ce $n$ 'est pas sans raison que I'imagerie $X$ mous n'a pu se développer que grâce à la forte luminance des sources synchrotrons.

* Par ailleurs, l'étendue de la source ne peut être inférieure à l'acceptance de l'optique sans modifier, parfois de façon importante les propriétés de l'image produite; c'est ce qu'on exprime en optique par la régle: "remplir la pupille du système".

Au total, on voit que la base principale de comparaison des sources est la luminance, associée à l'étendue (compte tenu de la méthode d'imagerie considérée). Le flux émis n'est jamais directement l'élément pertinent. La table ci-dessous donne les caractéristiques de quelques sources X typiques.

Tableau des caractéristiques de quelques sources $X$

\begin{tabular}{|c|c|c|c|}
\hline source & $\begin{array}{l}\text { flux } \\
\text { phot/s } \\
\text { ou /pulse }\end{array}$ & $\begin{array}{l}\text { émittance } \\
\text { (mm.mrad) }\end{array}$ & $\begin{array}{c}\text { luminance } \\
\left(\text { phot } /(\mathrm{mm} \cdot \mathrm{mrad})^{2} / \mathrm{s}\right. \\
\text { ou } / \text { pul se })\end{array}$ \\
\hline $\begin{array}{l}\text { Super ACO, SU7 } \\
(4,4 \mathrm{~nm}, 1 \% \text { BP })\end{array}$ & $210^{15}$ & $1 / 10^{-1}$ & $210^{16}$ \\
\hline $\begin{array}{c}\text { NSLS X1 } \\
(2,5 \mathrm{~nm}, 1 \% \mathrm{BP})\end{array}$ & $510^{15}$ & $10^{-1} / 210^{-3}$ & $510^{18}$ \\
\hline $\begin{array}{l}\text { Elettra (ondul.) } \\
(4,4 \mathrm{~nm}, 1 \% \mathrm{BP})\end{array}$ & $610^{14}$ & $10^{-2} / 210^{-3}$ & $310^{29}$ \\
\hline Tube X (microfoc.) & $10^{15}$ & $\approx 100$ & $10^{21}$ \\
\hline $\begin{array}{l}\text { plasma } X, C \text { DT: } 3,3 \mathrm{~nm} \\
\quad(1 \text { aser } \mathrm{KrF}, 100 \mathrm{hz})\end{array}$ & $10^{16}$ & $\approx 100$ & $10^{12} / \mathrm{s}$ \\
\hline 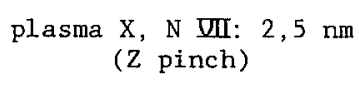 & $410^{14}$ & $\approx 100$ & $410^{20} / \mathrm{p}$ \\
\hline $\begin{array}{l}\text { source NL à champ } \\
\text { très intense }(\lambda=50 \mathrm{~nm})\end{array}$ & $510^{8} / \mathrm{p}$ & $10^{-2}$ & $510^{12} / \mathrm{p}$ \\
\hline laser $x, 10 \mathrm{~nm}$ & $10^{16} / \mathrm{p} ?$ & $10^{-3}$ & $10^{22} / \mathrm{p} ?$ \\
\hline
\end{tabular}

Pour terminer, il convient de signaler le lien profond qui existe entre les notions d'étendue et de cohérence spatiale.

En optique géométrique, un faisceau spatialement cohérent est un issu d'un point source: son étendue est donc nulle. En fait, on doit tenir compte de la diffraction, et de la limite de résolution qu'elle impose, d'où une étendue limite non nulle. Pour une source partiellement cohérente, c'est à dire dont 1 'étendue est nettement supérieure à la valeur limite, on peut définir une étendue cohérente, à l'intérieur de laquelle les rayons présentent une cohérence supérieure à un seuil donné. Sa valeur exacte dépend de la valeur du seuil, et du profil photométrique de la source, mais est toujours de $I^{\prime}$ ordre de $(\lambda / 2)^{2}\left(\lambda^{2} / 4 \pi\right.$ pour une source circulaire gaussienne). Le nombre de "cellule de cohérence" contenu dans l'étendue d'une source mesure son degré de cohérence; à $\lambda$ donné, c'est un invariant de la source au cours de la propagation du faisceau, au même titre que l'étendue.

\subsection{Les composants optiques $x$}

En $X$ comme dans le visible, la qualité des optiques peut être définie par:

- la qualité des surfaces d'onde produites, et l'importance des flux parasites (ordres, lumière diffuse); ces paramètres sont tout deux reliés à 1 'écart du composant à la forme idéale; 
- 1'efficacité énergétique;

- le domaine de longueur d'onde accepté.

Deux classes de composants $X$ sont utilisées en $X$ mous: les lentilles diffractives (réseaux zonés) et les miroirs.

\section{optiques diffractives}

Jusqu'à une époque très récente, i.l s'agissait uniquement de réseau zonés de Fresnel (RZF), improprement appellés "zones de Fresnel". Leur structure diffractante peut être absorbante ou déphasante. Leurs principales propriétés sont:

* le caractère multifocal: un RZF est équivalent à plusieurs lentilles de puissance croissant comme la suite des entiers. Ceci pose un problème de filtrage d'images parasites, en général résolu par un système de diaphragmes. On utilise usuellement l'ordre 1, parfois 1 'ordre 2 pour gagner en résolution. On note que du fait de 1 'équivalence RZF-1entille, la Ioi de conservation de l'étendue lui est applicable.

* Ie chromatisme: la puissance dépend de la longueur d'onde; il est donc nécessaire d'employer un rayonnement suffisamment monochromatique, ce qui est généralement obtenu à l'aide d'un RZF peu résolvant employé en monochromateur; de plus, la mise point doit être refaite après un changement de longueur d'onde;

* une efficacité (énergie dans l'ordre utile) limitée, qui dépend du matériau et de la longueur d'onde.

* la résolution permise, égale à la largeur du trait externe.

La qualité d'un RZF se mesure essentiellement aux erreurs sur le placement des traits du réseau. Dans la pratique, les erreurs sont locales et aléatoires, ce qui se traduit par la production d'une strrface d'onde diffuse, dont 1'énergie est complètement perdue. En d'autres termes (et contrairement aux miroirs) une optique diffractive non limitée par la diffraction (c'est à dire dont les zones externes sont de mauvaise qualité) ne procure aucun gain sur l'éclairement de 1'image, et dégrade même sa qualité.

Actuellement, les RZF les plus résolvants sont fabriqués par des techniques de microlithographie: tracé au masqueur électronique et gravure. Lorsque des épaisseurs importantes sont recherchées, il est nécessaire d'employer des techniques de transfert. Les meilleures zones obtenues à ce jour donnent une résolution de l'ordre de 30 à $40 \mathrm{~nm}$. La tendance observée est de réaliser des RZF "de phase" fonctionnant à des longueurs d'onde de plus en plus courtes (2,5 nm et inférieures). Il semble que la technique de fabrication des RZF butte sur un mur technologique vers $20-30 \mathrm{~nm}$.

\section{optiques à miroirs}

L'intérêt principal des optiques à miroirs est de permettre un fonctionnement géométrique, c'est à dire non limité par la diffraction. La résolution est alors limitée par les aberrations, et correspond à celle que donnerait une ouverture inférieure avec une optique 1imitée par la diffraction (DL). Mais l'acceptance correspond à l'ouverture réelle, d'où un éclairement image très supérieur. On parle actuellement d'ouvertures de $1^{\prime}$ ordre de 0,3 avec une résolution de $0,1 \mu \mathrm{m}$. A $\lambda=5 \mathrm{~nm}, 1$ 'ouverture DL correspondante n'est que 0,05 , soit un gain photonétrique de 1 'ordre de 36 sur une optique RZF de même résolution. De plus, une optique à miroirs est intrinsèquement non chromatique, c'est à dire que la position des images ne dépend pas de la longueur d'onde.

Malheureusement, 1 'efficacité énergétique des miroirs est conditionnée par leurs coefficients de réflection, qui en $X$, sont toujours très faibles en incidence normale. Ceci est d'autant plus gênant que la plupart des objectifs à miroirs utilisent au moins deux réflexions. Deux solutions sont donc possibles: travailler en incidence rasante sur des surfaces métallisées bien choisies, ou utiliser les traitements multicouches X.

La première solution, développée depuis longtemps, à conduit à la mise 
au point de formules optiques fondamentalement asphériques (Wolter) voire non de révolution (Kirckpatrick-Baez), et de formules dérivées. Ces formules ne permettent guère d'obtenir mieux que $1 \mu \mathrm{m}$ de résolution, mais elles conservent un certain intérêt en raison de leur efficacité énergétique. La deuxième solution implique la réalisation de multicouches efficaces; celles-ci sont obtenues en routine au dessus de $10 \mathrm{~nm}$, commencent à l'être entre 5 et $10 \mathrm{~nm}$, et ne le sont pas encore en dessous de $5 \mathrm{~nm}$. Sous réserve de la réalisation de couches efficaces, des optiques de type Schwarzschild (deux miroirs concentriques, obturation centrale) peuvent être utilisées, à condition de résoudre certaines difficultés supplémentaires, comme 1a variation des caractéristiques de couches en fonction de la distance à l'axe.

Outre le problème des couches réfléchissantes, la difficulté principale des optiques à miroirs réside dans la précision extrêmement élevée demandée à la foìs aux surfaces (en forme et rugosité, c'est à dire à toutes les fréquences spatiales), et au montage des miroirs. Ainsi, pour produire avec une optique à deux miroirs normaux une surface d'onde de forme meilleure que $\lambda_{x} / 2$ à $\lambda=5 \mathrm{~nm}$, on doit obtenir sur les surfaces une précision meilleure que $\lambda_{x} / 6$, soit référée à $\lambda_{w}=0,5 \mu \mathrm{m}, \lambda_{w} / 600$, et ce pour des formes sphériques, voire asphériques ! Nous pensons que ce défi technologique est susceptible de provoquer des progrès sensibles dans les techniques de contrôle et d'usinage des surfaces.

\section{optiques de Bragg-Fresnel}

Il s'agit d'optiques diffractives fonctionnant en réflexion, et basées sur une structure diffractante 3-D, par exemple multicouche $X$. Cette structure est déposée sur un substrat, qui peut être un plan, et de ce fait de très bonne qualité optique. Elle est ensuite gravée selon un réseau de Fresnel (ou autre) de façon à focaliser une surface d'onde stigmatique. Les premiers essais effectués ont donné une résolution de l'ordre de $2 \mu m$ à $\lambda=0,1 \mathrm{~nm}$, avec un système de type Kirkpatrick-Baez.

\section{IV - LES PRINCIPALES METHODES DE MICROSCOPIE X-MOUS}

Ce qui suit se veut un survol du domaine, destiné à donner des points de repère au lecteur non spécialiste. Pour une approche exhaustive, on pourra se reporter en particulier aux actes des colloques " $X$-ray microscopy" successifs (voir conclusion).

On peut regrouper les différentes méthodes en: microscopie de plein champ, microscopie à balayage, et microscopie sans optique $X$. Alors que les deux premières font usage d'optiques $X$, la dernière n'en utilise aucune, ce qui les rend technologiquement assez simples quant à leur partie strictement rayons $X$.

\section{1 la microscopie de plein champ}

On utilise un instrument d'optique au plein sens du terme, comportant un dispositif d'éclairage du plan objet (condenseur), et un "objectif" formant une image agrandie de l'objet. En principe, des optiques à miroir peuvent être employèes au même titre que des réseaux zonés. Toutefois, eu égard aux propriétés des optiques rasantes et au manque (sans doute provisoire) de multicouches efficaces en dessous de $50 \AA$, ce sont les RZF qui sont les plus utilisées, et qui ont atteints les performances les plus élevées. A ce jour, on atteint dans les meilleurs cas $30 \mathrm{rm}$ de résolution sur un champ de 30-40 $\mathrm{m}$, à une longueur d'onde de 2,5 à $4 \mathrm{~nm}$. Comme en microscopie classique, un fonctionnement en contraste de phase est possible, ce qui permet d'envisager un fonctionnement en dessous de $\lambda=2,5 \mathrm{~nm}$, ou au contraire au dessus du seuil du carbone (pour la biologie). Il est possible dans une certaine mesure de suivre un échantillon vivant (humide) en temps réel. Par contre, l'instrument est chromatique dans le cas des RZF, ce qui 
est un inconvénient dans la mesure ou un changement de longueur d'onde exige une refocalisation (ce qui $n^{\prime}$ est pas le cas des optiques à miroir, même à multicouches).

Les essais d'optiques à miroir utilisent les objectifs de Wolter ou de Schwarzschild, ces derniers étant subordonnés au progrès (d'ailleurs rapides) des traitements réfléchissant à multicouches X. On peut penser que le gain en flux autorisé par des optiques ouvertes correctement traitées, mêmes non limitées par la diffraction, donnera une impulsion nouvelle aux techniques de fabrication et d'assemblage des optiques à miroir à incidence normale.

En ce qui concerne les sources utilisables, c'est évidemment l'imagerie de plein champ qui est la moins exigeante en étendue, du fait du parallélisme élevé d'une image complète. A titre d'exemple et en terme d'émittance, pour un champ de $2 \times 15=30 \mu m$ ayant une résolution de $50 \mathrm{~nm}$ (1imite de diffraction), l'émittance acceptèe (au sens défini plus haut) est de 1'ordre de l'émittance correspondant à un pixel, c'est à dire l'émittance cohérente $\lambda / 2$ multipliée par le nombre de pixel soit 15/.05=300 dans l'exemple choisi. Elle est donc de $1^{\prime}$ ordre de $10^{-6} \mathrm{~m} . \mathrm{rad}$, ce qui est très élevé; et il est possible d'utiliser un aimant de courbure de synchrotron, voire même les sources X à plasma "de laboratoire" existantes.

\section{2 microscopie à balayage}

On forme sur l'échantillon l'image réduite d'un trou convenablement éclairé. L'échantillon est ensuite déplacé de façon à engendrer une image bidimensionnelle. Ce dispositif permet d'obtenir des images de transmission jusqu'à $0,1 \mu m$ de résolution. Il est possible d'obtenir un signal à partir d'objets de phase. Mais l'intérêt majeur réside dans la possibilité d'imagerie secondaire (voire multiple): fluorescence, photoémission.

Dans la mesure ou des optiques RZF sont utilisées, elles sont nécessairement limitées par la diffraction comme on l'a dit plus haut. Dans ce cas, on emploie une seule cellule de cohérence du faisceau $X$, ce qui, en $I^{\prime}$ état actuel, produit un signal détecté assez faible en imagerie de transmission, et très faible dans les modes secondaires, même avec le rayonnement synchrotron. C'est dire que les sources à plasma actuelles sont très insuffisantes, le laser $X$ étant impraticable du fait de son taux actuel de répétition. On comprend alors l'intérêt porté aux optiques à míroir à grande ouverture de type Schwarzschild, dans la mesure où une résolution analogue peut être obtenue.

\section{4,3 les microscopies sans optique $X$}

Ce sont essentiellement la microscopie de contact et l'holographie de Gabor. Ces deux méthodes ne font appel à aucune optique X dans la partie formation de l'image: de ce fait, aucune limitation technologique (ni aucun défaut d'image) provenant de la fabrication des optiques $X$ ne peut intervenir.

L'imagerie de contact consiste à placer l'échantillon directement sur le détecteur, usuellement photochimique (résine). C'est la plus ancienne des méthodes en rayons $X$, et de loin la plus simple et la plus résolvante (10 à $20 \mathrm{~nm}$ sur résine PMMA). G'est sans doute la raison pour laquelle elle reste utilisée par les biologistes. Sa difficulté essentielle reste 1'interprétation des images obtenues, surtout sur résine dans le cas des très haute résolution. En effet, d'une part les résines photosensibles employées sont fortement non linéaires, d'où un écrêtage de la dynamique par niveau insuffisant et saturation. D'autre part, la méthode ne procure aucun grandissement, ce qui impose une méthode de lecture ultra résolvante, usuellement la microscopie électronique à balayage après ombrage. Récemment, la microscopie à force atomique a été proposée comme outil de lecture.

La microscopie de contact est totalement non chromatique et utilise à peu près $n$ 'importe quel faisceau $X$, pourvi que l'éclairement soit suffisant 
pour assurer un temps de pose raisonnable. Sa seule limitation est liée à l'épaisseur de I'objet (défaut de contact), qui produit des franges de diffraction de Fresnel de dimension caractéristique $\sqrt{\lambda d}$. De plus, on ne peut obtenir qu'une image d'absorption.

La microscopie par photoémission est un moyen de surmonter la non linéarité du détecteur chimique: on remplace celui-ci par une photocathode, ce qui permet de transformer l'image d'absorption X en image d'émission électronique. Celle-ci peut alors être reprise et agrandie par un microscope électronique spécial. Ceci apporte 1'avantage d'une détection quantitative, 1inéaire, et en temps réel. Ce principe, proposé dès 1957 et étudié expérimentalement en 1985 , a été récemment repris par plusieurs laboratoires sous une forme légèrement modifiée: association d'un microscope $X$ de plein champ (haute résolution et faible grandissement, premier étage) et d'un étage "détecteur" à photoémission, à résolution moyenne et grandissement variable.

\section{L'holographie de Gabor}

Initialement proposée pour ses capacités d'imagerie $3-D$, il a été vite reconnu que celles-ci restent parfaitement théoriques tant que la résolution transverse obtenue est moins bonne que 2 à 3 longueurs d'onde. En fait, les arguments en faveur de l'holographie de Gabor sont de deux ordres: 1) simplicité: 1'opération d'enregistrement d'un hologramme n'est guère plus complexe que celle d'une image de contact, et permet même d'enregistrer un champ très important (plusieurs millimètres) quelle que soit la résolution finale. 2) nature du signal: par nature, l'hologramme enregistre simultanément la phase et l'amplitude de l'objet.

La difficulté principale de l'holographie est l'étape dite de reconstruction, qui permet de former une image de l'objet à partir de l'hologramme. Ceci peut être effectué soit par ordinateur, après numérisation de l'hologramme, soit par voie optique en utilisant une source UV. Dans le cas numérique (résolution rapportée $0,1 \mu \mathrm{m}$ ), la limite accessible dans un futur prévisible est probablement proche de $20 \mathrm{~nm}$, à condition de mettre au point un outil spécifique de numérisation des hologrammes. Dans le cas optique (projet en cours pour une résolution de $0,09 \mu \mathrm{m}$ ), la résolution accessible est probablement limitée à 50-60 nm, mais cette méthode offre l'avantage d'un instrument de reconstruction dédié, semblable à un microscope optique, qui peut être d'un emploi particulièrement convivial.

On sait que l'holographie est une méthode interférométrique, ce qui pose le problème de la cohérence du faisceau X. En fait, grâce à l'enregistrement parallèle de tous les points du champ et aux propriétés particulières de l'holographie de Gabor (cohérence spatiale locale), la cohérence nécessaire est nettement inférieure à celle exigée par la microscopie à balayage. On peut montrer qu'il existe pour des conditions d'enregistrement données et un champ donné, une émittance optimale de faisceau. Celle ci peut correspondre dans des conditions standards d'enregistrement à quelques dizaines d'émittances cohérentes.

\section{V - CONCLUSION et REFERENCES}

Nous avons brièvement présenté les techniques et les méthodes utilisées en microscopie $X$-mous, ainsi que les paramètres qui permettent de comparer dans ce contexte les sources $X$ employées. Initialement étudiées en vue d'application à la biologie, les techniques développées sont maintenant susceptibles de déborder largement ce cadre.

Des informations plus détaillées, ainsi que des images obtenues par les diverses méthodes présentées, peuvent être trouvées dans la littérature. On peut en particulier se reporter aux ouvrages suivants:

"X-ray microscopy III", A.G. Michette, G.R. Morrison et C.J. Buckley eds, Springer (Berlin, 1992).

A.G. Michette, "Optical systems for soft X-rays", Plenum Press (1986). 